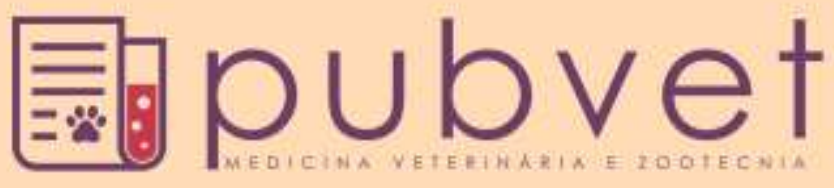

https://doi.org/10.22256/pubvet.v12n2a25.1-7

\title{
Sarcóide recidivante em glande de um equino: Relato de caso
}

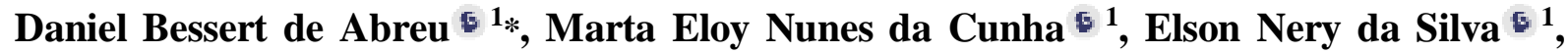 \\ Roberto Viana Menezes ${ }^{\sqrt{6}}$, Margareth Moura Ferreira ${ }^{62}$, Anderson Luiz de Araújo ${ }^{\sqrt{6}}$, \\ Maria Consuêlo Caribé Ayres 5
}

\footnotetext{
${ }^{1}$ Médico(a) Veterinário(a) Residente em Clinica de Ruminantes e Equídeos, Universidade Federal da Bahia, Salvador, Brasil

${ }^{2}$ Médico (a) Veterinário do Centro de Desenvolvimento da Pecuária, Universidade Federal da Bahia, Santo Amaro, Brasil

${ }^{3}$ Médico Veterinário do Hospital Veterinário, Universidade Federal da Bahia, Salvador, Bahia, Brasil

${ }^{4}$ Prof $^{a}$ do Departamento de Anatomia, Patologia e Clínicas, Universidade Federal da Bahia, Salvador, Bahia, Brasil.

*Autor para correspondência, E-mail: danielbessert@hotmail.com
}

RESUMO. Sarcóides são tumores de pele do tipo fibroblásticos não metastatizantes, considerados os mais comuns em equinos. Sua etiopatogenia está relacionada à infecção pelo papilomavírus bovino do tipo 1 e tipo 2 . A transmissão e a disseminação para outras partes do corpo do mesmo animal estão associadas a mordidas, fricção, fômites e insetos. Apresentam-se agressivos no local afetado devido à capacidade infiltrativa e geralmente refratários às diferentes formas de terapias. Os sarcóides são classificados em seis formas de acordo com as suas apresentações clínicas, sendo estas: oculto, verrucoso, nodular, fibroblástico, misto e maligno. O objetivo do presente trabalho foi relatar o caso clínico de sarcóide recidivante localizado na glande de um equino atendido na Clínica de Equídeos do Centro de Desenvolvimento da Pecuária da Universidade Federal da Bahia, situado no município de Santo Amaro - BA. No presente caso clínico, um equino apresentou uma massa tumoral na região da glande, onde foi realizada a éxerese da massa e através de exame histopatológico observou-se características compatíveis com sarcóide. Após aproximadamente quatro meses da intervenção cirúrgica, o sarcóide apresentou recidiva sendo instituído tratamento com associação entre penectomia parcial, pomada a base de aciclovir a $5 \%$ e auto-hemoterapia

Palavras chave: cavalo, tumor, penectomia, aciclovir.

\section{Recurrent sarcoid in the glans of an equine: Case report}

\begin{abstract}
Sarcoids are fibroblast and non-metastatic skin tumors, representing the most common tumors in horses. Its etiopathogenesis is related to bovine papillomavirus type 1 and type 2 infections, being the transmission and dissemination to other parts of the body of the same animal associated with bites, friction, fomites and insects. Clinical behavior is locally aggressive due to infiltrative capacity, as well as being refractory to different forms of therapies. The sarcoids are classified in six forms according to their clinical presentations, being these: hidden, verrucous, nodular, fibroblastic, mixed and malignant. The objective of this study is to report a clinical case of recurrent sarcoid in the equine glans, attended at the equine clinic of the Centro de Desenvolvimento da Pecuária of the Universidade Federal da Bahia, located in the municipality of Santo Amaro - BA. In the present case, an equine presented a tumor mass in the region of the glans, where a mass examination and histopathological examination were carried out; characteristics compatible with the sarcoid were observed. After five months of surgical intervention, the sarcoid presented recurrence with treatment instituted with an association between partial penectomy, ointment based on acyclovir 5\% and autohemotherapy.
\end{abstract}


Keywords: horse, tumor, penectomy, acyclovir

\title{
Sarcoide recidivante en glande de un equino: Reporte de un caso
}

\begin{abstract}
RESUMEN. Los sarcoides son tumores de piel del tipo fibroblásticos, sin metástasis, considerados los más comunes en equinos. Su etiopatogenia está relacionada con la infección por el papilomavirus bovino del tipo 1 y el tipo 2. La transmisión y la diseminación a otras partes del cuerpo del mismo animal están asociadas a mordidas, fricción, fómites e insectos. Se presentan agresivos en el lugar afectado debido a la capacidad infiltrativa y generalmente refractarios a las diferentes formas de terapias. Los sarcoides se clasifican en seis formas de acuerdo con sus presentaciones clínicas, siendo estas: oculto, verrugoso, nodular, fibroblástico, mixto y maligno. El objetivo del presente trabajo fue relatar el caso clínico de sarcóide recidivante localizado en el glande de un equino atendido en la Clínica de Équidos del Centro de Desarrollo de la Pecuaria de la Universidade Federal de Bahía, situado en el municipio de Santo Amaro - BA . En el presente caso clínico, un equino presentó una masa tumoral en la región del glande, donde fue realizada la extirpación quirúrgica de la masa y a través de examen histopatológico se observaron características compatibles con sarcoide. Después de aproximadamente cuatro meses de la intervención quirúrgica, el sarcoide presentó recidiva siendo instituido tratamiento con asociación entre penectomía parcial, pomada a base de Aciclovir al 5\% y auto-hemoterapia
\end{abstract}

Palabras clave: caballo, tumor, penectomía, Aciclovir

\section{Introdução}

Sarcóides são tumores de pele do tipo fibroblásticos e não metastatizantes, representando os tumores mais comuns em equídeos e, geralmente, acomete animais com idade inferior a quatro anos, independentemente da raça, do sexo ou mesmo das características de pelagens, apresentando distribuição cosmopolita (Theón, 2007). Sua etiopatogenia está relacionada à infecção pelo papilomavírus bovino (PVB) tipo 1 , considerado mais comum e tipo 2 , sendo a transmissão e disseminação para outras parte do corpo do mesmo animal associada às mordidas, fricção, fômites e insetos. A apresentação clínica é caracterizada por lesões locais e agressivas, desenvolvendo-se frequentemente em membros, cabeça, região periorbital e palpebral, região ventral do abdômen, base da orelha, região axilar e inguinal, ou seja, regiões de pele fina e pouco pêlo. Pode surgir de forma múltipla em várias partes do corpo ou com uma única lesão inicial de aspecto verrucoso. Estas lesões podem desenvolver fibrose, tornando-as firme à palpação, e aumentando consideravelmente de tamanho, além de em alguns casos apresentar ulceração das lesões com consequente infecção bacteriana e inflamação de áreas periféricas. Devido a esta variabilidade no desenvolvimento das lesões e ao potencial de transformação destas em tipos clínicos diferentes dos iniciais durante as frequentes recidivas, o sarcóide geralmente é refratário às diferentes formas de terapias (Thomassian, 2005, Cremasco \& Sequeira, 2010, Taylor \& Haldorson, 2013, Theón, 2007).

Os sarcóides são classificados em seis formas de acordo com as suas apresentações clínicas, sendo estas: oculto, verrucoso, nodular, fibroblástico, misto e maligno. O tipo oculto é caracterizado por áreas circulares de alopecia e rugosidade na pele. $\mathrm{O}$ tipo verrucoso tem aparência semelhante a uma verruga. $O$ tipo nodular apresenta lesões subcutâneas bem definidas, apresentando lóbulos únicos ou agregados. O sarcóide tipo fibroblástico possui aparência exofítica fibrovascular e ulcerada, frequentemente lembrando tecido de granulação. O sarcóide do tipo maligno é invasivo apresentando infiltração linfática e vascular local, porém não metastático. Os tipos mistos são também reconhecidos como formas relativamente comuns e representam lesões que possuem características pertencentes a dois ou mais tipos descritos anteriormente (Cremasco \& Sequeira, 2010, Radostits et al., 2010).

Os diagnósticos diferencias para sarcóide equino incluem tecido de granulação exuberante, papiloma, fibroma, fibrossarcoma, linfoma cutâneo, carcinoma de células escamosas, habronemose, melanoma e foliculites por Staphylococcos sp. O diagnóstico baseia-se nos dados epidemiológicos, sinais clínicos e pelo 
exame histopatológico. Histopatologicamente, o sarcóide é caracterizado por marcada proliferação dérmica de fibroblastos, orientação perpendicular dos fibroblastos da junção dermo-epidérmica relação à membrana basal e hiperplasia epidérmica com projeções epiteliais em direção a derme (Foy et al., 2002).

O objetivo do presente trabalho foi relatar um caso clínico de sarcóide recidivante na glande de equino, atendido na clínica de equídeos do Centro de Desenvolvimento da Pecuária (CDP) da Universidade Federal da Bahia (UFBA), situado no município de Santo Amaro - BA.

\section{Relato do caso}

Em setembro de 2015, um equino macho, castrado, de pelagem castanha, sem raça definida, com 15 anos de idade foi encaminhado a Clinica de Equídeos do Centro de Desenvolvimento da Pecuária, pertencente à Universidade Federal da Bahia. O animal apresentava um aumento de volume, com características tumorais na região crânio-dorsal da glande. Segundo o proprietário, a lesão iniciou-se cerca de seis meses antes, observando-se pequenos nódulos firmes à palpação e não ulcerados. Todavia, não havia histórico de trauma na região. $O$ animal era mantido em pastagem de Brachiaria decumbens, sem contato com outros equídeos. Entretanto, havia contato direto com bovinos, os quais não apresentavam nenhuma enfermidade. Ao exame físico, o animal encontrava-se com escore de condição corporal $3 / 5$, peso corpóreo de $450 \mathrm{~kg}$, sem alterações nos padrões fisiológicos para a espécie e idade conforme Riet-Correa et al. (2007). Os valores dos componentes do hemograma dentro dos limites dos valores de referência para a espécie (Tabela 1). A massa tumoral possuía área de aproximadamente $10 \mathrm{~cm}^{3}$, localizando-se de forma circunscrita na região crânio dorsal da glande, que apresentava áreas ulceradas além da presença de miíases (Figura 1). Devido ao histórico, localização da lesão e apresentação clínica, a suspeita inicial foi de carcinoma de células escamosas. Assim, a terapia instituída foi à excisão cirúrgica total da massa tumoral.

Para a realização do procedimento cirúrgico, o animal foi submetido à anestesia intravenosa total, sendo utilizado como medicação pré-anestésica acepromazina $(0,03 \mathrm{mg} / \mathrm{kg} / \mathrm{IV})$ e xilazina $(0,8$ $\mathrm{mg} / \mathrm{kg} / \mathrm{IV})$. A indução foi realizada com cetamina $(2,0 \mathrm{mg} / \mathrm{kg} / \mathrm{IV})$ e midazolam $(0,1 \mathrm{mg} / \mathrm{kg} / \mathrm{IV})$ e a manutenção com "triple drip" (2 g cetamina, 500 mg xilazina e 1 litro de EGG à 5\%/IV) na taxa de infusão de $3 \mathrm{ml} / \mathrm{kg} / \mathrm{h}$. A massa tumoral foi removida após diérese circular desta com margens de segurança de um centímetro, utilizando lâmina de bisturi $\mathrm{n}^{\circ} 22$ e posterior síntese e reconstrução da glande utilizando fio catgut $\mathrm{n}^{\circ} 0$. Para a hemostasia foi realizado compressão proximal no pênis com fita elástica de látex. Após a remoção da massa tumoral, o animal permaneceu internado por cinco dias. Neste período foi realizada a antibioticoterapia (enrofloxacina, na dose de 4 $\mathrm{mg} / \mathrm{kg}$, por via intramuscular, a cada 24 horas, por 5 dias), terapia anti-inflamatória (flunixin meglumine, na dose de $1,1 \mathrm{mg} / \mathrm{kg}$, por via intramuscular, a cada 24 horas, por 3 dias) e curativo diário da ferida operatória utilizando solução alcoólica de clorexidina a $0,5 \%$ e solução salina $(\mathrm{NaCl} 0,9 \%)$. Após cinco dias, o animal apresentou boa evolução da ferida operatória não sendo observado deiscência da sutura e edema adjacente a área.

Tabela 1. Valores hematológicos no momento do primeiro atendimento em setembro de 2015.

\begin{tabular}{lcc}
\hline & $\begin{array}{c}\text { Valores } \\
\text { Encontrados }\end{array}$ & $\begin{array}{c}\text { Valores de Referência, } \\
\text { Kaneko et al. (2008) }\end{array}$ \\
\hline Hematócrito, \% & 35,0 & $32,0-53,0$ \\
Hemácias, x10\% $\mu \mathrm{L}$ & 8,6 & $6,8-12,9$ \\
Hemoglobina, g/dL & 11,6 & $11,0-19,0$ \\
VGM, fL & 40,6 & $37,0-59,0$ \\
CHGM, \% & 33,1 & $31,0-38,6$ \\
PPT, g/dL & 7,0 & $6,0-7,7$ \\
Fibrinogênio & 200 & $100-400$ \\
Leucócitos, / $\mu \mathrm{l}$ & 7.000 & $5.400-14.300$ \\
Segmentados & 2.940 & $2.300-6.500$ \\
Eosinófilos & 210 & $0-1000$ \\
Linfócitos & 3.430 & $1.500-7.700$ \\
Monócitos & 420 & $0-1000$ \\
\hline
\end{tabular}

O tecido retirado no procedimento foi acondicionado e fixado em formol a $10 \%$ e encaminhado para o Laboratório de Patologia Veterinária da UFBA. No exame histopatológico, constatou-se que o material apresentava características compatíveis com sarcóide equino, apresentando proliferação fibroblástica bem diferenciada, bem como hiperplasia pseudoepiteliomatosa, acantose e hiperqueratose ortoqueratótica. Devido à dificuldade de localização e comunicação com o proprietário, os demais tratamentos recomendados para sarcóide não puderam ser realizados. 


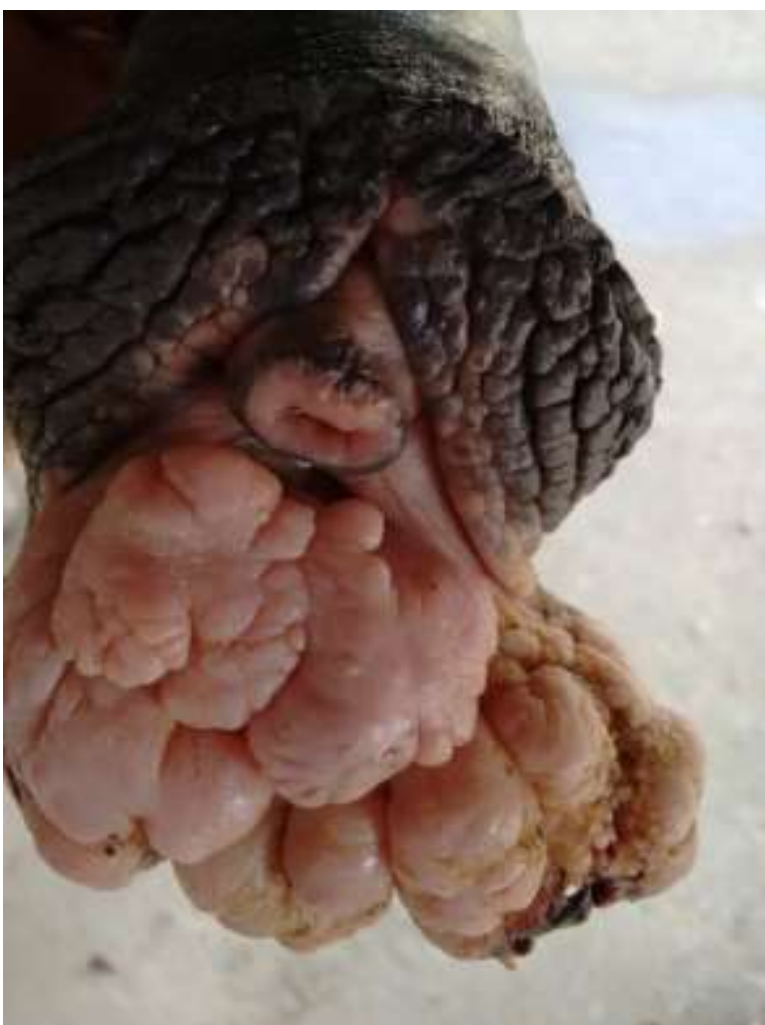

Figura 1. Massa tumoral na região da glande de um equino.

Após aproximadamente um ano, o animal retornou a Clínica de Equídeos do CDP-UFBA apresentando nova massa tumoral na região da glande, de crescimento progressivo e surgimento cerca de quatro meses após a intervenção cirúrgica. Ao exame clínico, o animal apresentava-se caquético, pesando $250 \mathrm{~kg}$, com alta infestação por carrapatos (Amblyomma sp. e Dermacentor sp.), normotermia $\left(37,8^{\circ}\right.$ C) moderada anemia normocitica normocrômica, leucograma dentro dos valores de referência (Tabela 2). A massa tumoral possuía área de aproximadamente $15 \mathrm{~cm}^{3}$, localizava-se de forma circunscrita ocupando toda a superfície da glande e não apresentava áreas ulceradas. Devido ao péssimo estado clínico, foi decidida pela internação do animal. Um novo procedimento cirúrgico foi realizado, sendo desta vez optada pela penectomia segundo técnica descrita por Williams \& Williams (1943). O protocolo anestésico utilizado foi o mesmo descrito anteriormente para a realização do primeiro procedimento cirúrgico. Assim como instituído no primeiro internamento do animal foi realizada antibioticoterapia (ceftiofur, na dose de $2,2 \mathrm{mg} / \mathrm{kg}$, por via intramuscular, a cada 24 horas, por 7 dias), terapia antiinflamatória (flunixin meglumine, na dose de $1,1 \mathrm{mg} / \mathrm{kg}$, por via intramuscular, a cada 24 horas, por 3 dias), curativo diário da ferida operatória utilizando solução aquosa de clorexidina a $1 \%$ e solução salina $(\mathrm{NaCl} 0,9 \%)$, após sete dias da cirurgia o animal apresentou boa evolução da ferida operatória, não sendo observado deiscência da sutura e edema adjacente a área. Devido ao resultado do primeiro exame histopatológico foi estabelecido tratamento tópico pós-cirúrgico com pomada a base de aciclovir a $5 \%$ em todo terço final do pênis a cada 48 horas durante 30 dias e auto-hemoterapia conforme descrito por Thomassian (2005). Para exposição e manuseio do pênis, era realizado bloqueio perineural do nervo pudendo no nível do arco isquiático, utilizando para tal $2,5 \mathrm{ml}$ de lidocaína a $2 \%$ em cada antímero. Após nova avaliação histopatológica, foi confirmada a presença do sarcóide recidivante. O material enviado para exame histopatológico apresentava marcada proliferação dérmica fibroblástica associada a fibras colágenas, e presença de neovascularização intensa, fibroblastos dispostos de forma irregular formando feixes alongados entrelaçados e em espiral, hiperplasia epidérmica irregular contendo projeções digitiformes orientadas para o interior da derme (Figura 2A e 2B).

Tabela 2. Valores hematológicos no momento do segundo atendimento, aproximadamente um ano após o primeiro

\begin{tabular}{lcc}
\hline & $\begin{array}{c}\text { Valores } \\
\text { Encontrados }\end{array}$ & $\begin{array}{c}\text { Valores de Referência } \\
\text { Kaneko et al. (2008) }\end{array}$ \\
\hline Hematócrito, \% & 17,0 & $32,0-53,0$ \\
Hemácias, x10\%/ $\mu \mathrm{L}$ & 4,3 & $6,8-12,9$ \\
Hemoglobina, g/dL & 5,7 & $11,0-19,0$ \\
VGM, fL & 39,5 & $37,0-59,0$ \\
CHGM, \% & 33,5 & $31,0-38,6$ \\
PPT, g/dL & 6,0 & $6,0-7,7$ \\
Fibrinogênio, mg/dL & 200 & $100-400$ \\
Leucócitos, / $\mu$ l & 6.700 & $5.400-14.300$ \\
Segmentados & 2.345 & $2.300-6.500$ \\
Eosinófilos & 402 & $0-1000$ \\
Linfócitos & 3.752 & $1.500-7.700$ \\
Monócitos & 201 & $0-1000$ \\
\hline
\end{tabular}

O animal foi acompanhado por 90 dias após a intervenção cirúrgica. Após este período, não foi observado reaparecimento de nenhuma massa neoplásica na região do pênis ou em outra região. 


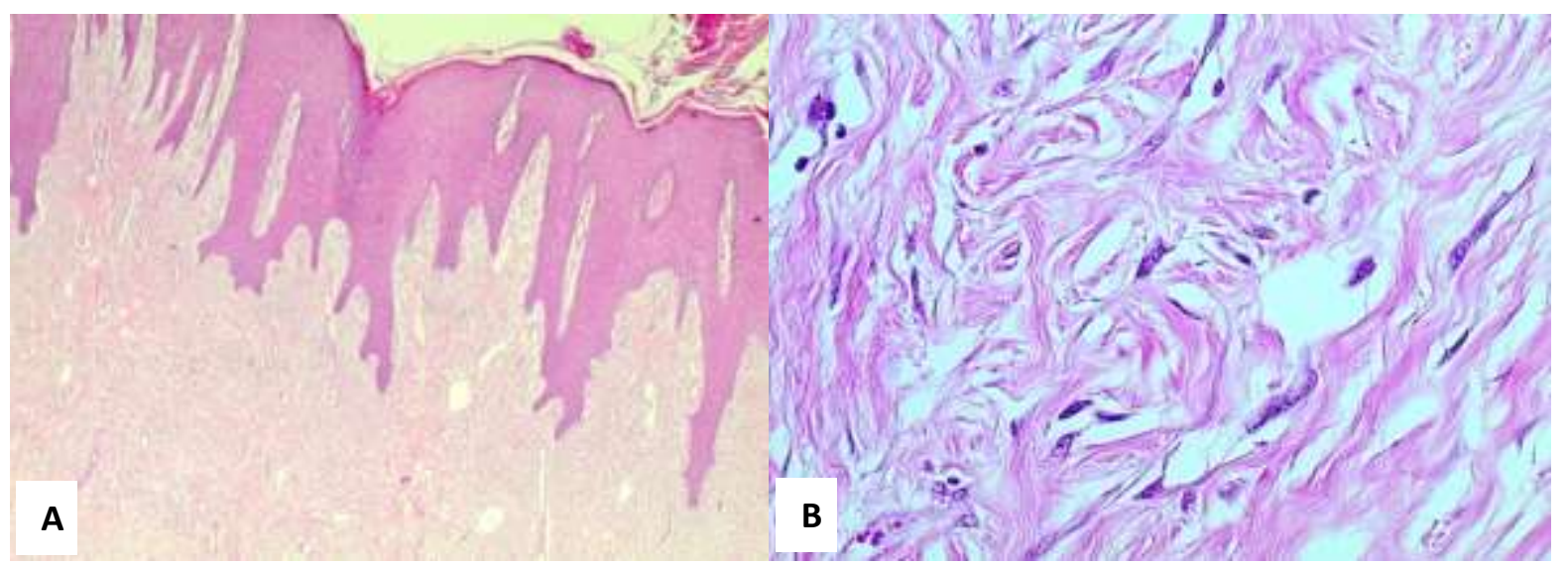

Figura 2. A. Hiperplasia epidérmica irregular contendo projeções digitiformes orientadas para o interior da derme. Aumento de 4x. B. Marcada proliferação dérmica fibroblástica associada a fibras colágenas, e presença de neovascularização intensa, fibroblastos dispostos de forma irregular formando feixes alongados entrelaçados e em espiral. Aumento de 40x.

\section{Discussão}

Dentre as neoplasias que acometem equídeos, o sarcóide é o que apresenta maior incidência (Theón, 2007). Em estudo realizado por Souza et al. (2009), onde foram avaliadas amostras de tumores cutâneos recebidas no Laboratório de Patologia Veterinária da Universidade Federal de Santa Maria, constatou-se que 62/108 (57,4\%) das amostras eram de sarcóide equino. Carvalho et al. (2014) realizaram um estudo retrospectivo das neoplasias em ruminantes e equídeos no semiárido do nordeste brasileiro, no qual observaram $45,3 \%$ (29/64) de amostras compatíveis com sarcóide. Baseado nesses estudos, provavelmente, a ocorrência do sarcóide equino sofre uma variação regional.

Embora o sarcóide seja considerado a neoplasia cutânea de maior incidência em equídeos, o mesmo não é constantemente relatado na região de pênis e prepúcio (Knottenbelt et al., 1995, Semieka et al., 2012), conforme observado no presente relato.

Em estudo retrospectivo realizado por Van den Top et al. (2008), no qual avaliaram os achados clínicos e histopatológicos de 114 equinos machos acometidos por tumores na genitália externa, observou-se que nenhum equino foi acometido por sarcóide. Entretanto, em amostras neoplásicas obtidas da região de pênis e prepúcio foi constatada a incidência de sarcóide igual a 10,3\% (3/29), sendo que nenhuma dessas amostras foi proveniente da região da glande como o relatado no presente trabalho ( $\underline{\text { Xavier, 2010) }}$.

Para a instituição do tratamento alguns fatores devem ser levados em consideração. Dentre eles, o valor financeiro do animal e do tratamento, o local da lesão, tratamentos prévios, histórico da lesão, existência de tecido de granulação ou outra neoplasia concomitante e praticidade do tratamento a ser estabelecido. Inúmeros tratamentos já foram descritos para o sarcóide equino, tais como tratamentos cirúrgicos, incluindo a exérese da massa tumoral usada no presente relato, criocirugia e cirurgia a laser e tratamentos não cirúrgicos, como ligadura, quimioterapia, radioterapia e imunoterapia (Bogaert et al., 2008, Knottenbelt et al., 1995, Taylor \& Haldorson, 2013). No presente relato, foi feita a opção única pelo tratamento cirúrgico no primeiro encaminhamento do animal devido a suspeita inicial de carcinoma de células escamosas, o valor financeiro do animal e o alto custo de outras terapias como a quimioterapia e a radioterapia.

Entretanto, não houve uma evolução satisfatória após a exérese da massa tumoral no equino estudado, com recidiva a qual ocorreu após quatro meses, sendo que o mesmo só retornou para um novo tratamento um ano após a ocorrência do primeiro episódio. Segundo a literatura, a exérese da massa tumoral possui uma taxa de sucesso variada, apresentando recidiva em torno de 40 a $72 \%$, sendo que este reaparecimento pode ocorrer em até cinco anos. Isto ocorre uma vez que o PVB encontra-se latente na pele integra e após o trauma cirúrgico pode ser ativado, alem do fato de ocorrer auto transplante das células tumorais durante o procedimento. Tumores recorrentes são mais agressivos e crescem mais rapidamente que os tumores iniciais. A fim de evitar o reaparecimento do tumor a associação com outras técnicas tais como criocirurgia, quimioterapia e imunoterapia diminuem a frequência da recidiva (Ragland et al., 1970, Diehl et al., 1987, Broström, 1995, Bogaert et al., 2008, Knottenbelt et al., 1995). 
O tratamento de sarcóide com imunoterápicos tem sido amplamente utilizado, os mais comumente utilizados são o Bacilo de Calmette e Guerin (BCG) intralesional, vacinação autógena, imiquimode tópico e aciclovir tópico (Radostits et al., 2010, Taylor \& Haldorson, 2013). Sendo assim, para evitar uma nova recidiva, a proposta terapêutica no retorno do referido animal incluiu associação do procedimento cirúrgico com o uso de imunoterápicos.

O uso tópico de aciclovir tem sido um tratamento promissor, pois apresenta bons resultados, é de fácil utilização e tem baixo custo quando comparado a outras terapias, como a BCG (Knottenbelt et al., 1995, Bromerschenkel \& Figueiró, 2013). Stadler et al. (2011) utilizaram o creme de aciclovir a 5\% em 47 lesões de sarcóide equino, diariamente durante dois a seis meses. Ao término do experimento, todos os 47 tumores responderam satisfatoriamente ao tratamento, sendo que a regressão completa ocorreu em $68 \%$ (32/47) das lesões. A falha no tratamento com aciclovir pode estar proporcionalmente associada à espessura da lesão, sugerindo que a droga possui habilidade moderada para penetrar em áreas mais profundas. Portanto, recomenda-se a exérese de massas tumorais maiores que $5 \mathrm{~mm}$ de diâmetro com posterior utilização do creme de aciclovir a $5 \%$ sobre a lesão (Stadler et al., 2011, Taylor \& Haldorson, 2013), conforme feito no segundo protocolo terapêutico estabelecido no presente relato.

A auto-hemoterapia, adotada como terapia complementar no presente estudo, é uma técnica que tem como intuito modular os fatores de crescimento e anticorpos, sendo bastante difundidas por possui valor terapêuticas em afecções alérgicas, desordens circulatórias, afecções virais e em crescimentos neoplásicos. Todavia, existe a necessidade de maiores estudos da sua eficácia no tratamento de sarcóide equino. A técnica consiste em administrar $10 \mathrm{~mL}$ de sangue venoso do próprio animal pela via intramuscular, uma vez a cada sete dias, totalizando quatro aplicações (Thomassian, 2005, Sánchez-Casanova et al., 2015). A opção pela penectomia parcial, seguindo a técnica descrita por Williams \& Williams (1943) foi feita devido à recidiva, localização e tamanho relativamente grande da massa tumoral, desta forma dificultando a realização de outros tipos de tratamentos. De acordo com Rizk et al. (2013), a realização da penectomia parcial é indicada em neoplasias que acometem o terço distal do pênis, tendo em vista a baixa ocorrência de recidivas e complicações como a estenose uretral.

\section{Conclusões}

A partir do presente relato, foi possível observar que o tratamento para o sarcóide equino localizado na glande baseado somente na exérese da massa tumoral não se mostrou satisfatório, com a lesão recidivando. Tal lesão, só foi controlada, em um segundo atendimento veterinário, ao associar à técnica de penectomia parcial com uma terapia imunomoduladora a base de aciclovir a 5\% e auto-hemoterapia.

\section{Referências Bibliográficas}

Bogaert, L., Martens, A., Depoorter, P. \& Gasthuys, F. 2008. Equine sarcoids, part 2: Current treatment modalities. Vlaams Diergeneeskundig Tijdschrift, 77, 62-67.

Bromerschenkel, I. \& Figueiró, G. M. 2013. Tratamentos do sarcóide equino. Agropecuária Científica no Semiárido, 9, 07-10.

Broström, H. 1995. Equine sarcoids. A clinical and epidemiological study in relation to equine leucocyte antigens (ELA). Acta Veterinaria Scandinavica, 36, 223-236.

Carvalho, F. K. L., Dantas, A. F. M., Riet-Correa, F., Andrade, R. L. F. S., Nóbrega Neto, P., Miranda Neto, E., Simões, S. V. D. \& Azevedo, S. S. 2014. Estudo retrospectivo das neoplasias em ruminantes e equídeos no semiárido do Nordeste Brasileiro. Pesquisa Veterinária Brasileira, 34, 211-216.

Cremasco, A. C. M. \& Sequeira, J. L. 2010. Sarcóide equino: aspectos clínicos, etiológicos e anatomopatológicos. Veterinária e Zootecnia, 17, 191-199.

Diehl, M., Vingerhoets, M. \& Stornetta, D. 1987. Spezifische methoden zur entfernung des equinen sarkoides. Der Praktische Tierarzt, Collegium Veterinarium, 18, 14-16.

Foy, J. M., Rasshimir, Raven, A. M. \& Brasshier, M. K. 2002. Common equine skin tumors. Compendium on Continuing Education for the Practicing Veterinarian, 24, 242.

Kaneko, J. J., Harvey, J. W. \& Bruss, M. L. 2008. Clinical biochemistry of domestic animals. Academic press, New York.

Knottenbelt, D., Edwards, S. \& Daniel, E. 1995. Diagnosis and treatment of the equine sarcoid. In Practice, 17, 123-129. 
Radostits, O. M., Gay, C. C., Blood, D. C., Hinchcliff, K. W. \& McKenzie, R. A. 2010. Clínica Veterinária: um tratado de doenças dos bovinos, ovinos, suínos, caprinos $e$ eqüinos. Guanabara Koogan, Rio de Janeiro.

Ragland, W. L., Keown, G. H. \& Spencer, G. R. 1970. Equine sarcoid. Equine Veterinary Journal, 2, 2-11.

Riet-Correa, F., Méndez, F., Carmen Schild, M., Riet-Correa, A. L. F., Schild, A. L. \& Carmen, M. M. 2007. Doenças de ruminantes e eqüinos. Varela, Sãp Paulo.

Rizk, A., Mosbah, E., Karrouf, G. \& Abou Alsoud, M. 2013. Surgical management of penile and preputial neoplasms in equine with special reference to partial phallectomy. Journal of Veterinary Medicine, 2013, 1-8.

Sánchez-Casanova, R., Masri-Daba, M., AlonsoDíaz, M., Méndez-Bernal, A., Hernández-Gil, M. \& Fernando-Martinez, J. 2015. Ethnoveterinary practices used by owners of working equids with dermal disorders from tropical regions of Veracruz, Mexico. Journal of Traditional knowledge, 14, 344-350.

Semieka, M. A., Ali, M. M. \& Al-Lethie, A. A. 2012. Sarcoids in donkeys: common types and available treatment. Journal of Advanced Veterinary Research, 2, 276-283.

Souza, T. M., Fighera, R. A., Schmidt, C., Réquia, A. H., Brum, J. S., Martins, T. B. \& Barros, C. S. L. 2009. Prevalência das dermatopatias nãotumorais em cães do município de Santa Maria, Rio Grande do Sul (2005-2008). Pesquisa Veterinaria Brasileira, 29, 157-162.
Stadler, S., Kainzbauer, C., Haralambus, R., Brehm, W., Hainisch, E. \& Brandt, S. 2011. Successful treatment of equine sarcoids by topical aciclovir application. The Veterinary Record, 168, 187.

Taylor, S. \& Haldorson, G. 2013. A review of equine sarcoid. Equine Veterinary Education, 25, 210-216.

Theón, P. A. 2007. Equine Sarcoid. In: Smith, P. (ed.) Large Animal Internal Medicine. Elsevier, Saint Louis, USA.

Thomassian, A. 2005. Enfermidades dos cavalos. 4 ed. Sao Paulo: Livraria Varela, 2005. cap 2, p 42-43. Livraria Varela, Sao Paulo, Brasil.

Van den Top, J. G. B. v. d., Heer, N., Klein, W. R. \& Ensink, J. M. 2008. Penile and preputial tumours in the horse: a retrospective study of 114 affected horses. Equine Veterinary Journal, 40, 528-532.

Williams, W. L. \& Williams, W. W. 1943. The diseases of the genital organs of domestic animals. The Author.

Xavier, F. S. 2010. Lesões proliferativas de pênis e prepúcio eqüinos. Faculdade de Veterinária. Universidade Federal de Pelotas, Pelotas, Rio Grande do Sul.

\section{Article History:}

Received 29 September 2017

Accepted 27 October 2017

Available online 4 January 2018

License information: This is an open-access article distributed under the terms of the Creative Commons Attribution License 4.0, which permits unrestricted use, distribution, and reproduction in any medium, provided the original work is properly cited. 